\title{
Christa Wichterich
}

\section{Anerkennung, Rechte, Ressourcen Frauenorganisationen im informellen Sektor in Ländern des Südens}

Informeller Sektor und Organisierung erscheinen als Widerspruch in sich. Eine der vielen Definitionen des informellen Sektors ist, dass er nicht kollektiven Aushandlungsprozessen unterworfen ist (Hosmer Martens/Mitter 1994:6). Seine vereinzelnden Arbeitsformen und häufig wechselnden Beschäftigungsverhältnisse scheinen schwer oder gar unüberwindbare Hindernisse für Solidarisierung und Organisierung zu sein. Doch seit den siebziger Jahren sind neue Organisationen informell Tätiger entstanden, vor allem von Frauen.

Diese Selbstorganisierung ist als Prozess selbstbestimmter, interessengeleiteter Vergesellschaftung zu verstehen. Sie hat die Vergesellschaftung und Vergeschlechtlichung durch Arbeit im informellen Sektor als Grundlage und ihre Veränderung als Ziel. Ihre Organisations- und Aktionsformen, ihre Konzepte und Strategien sind mit konventionellen gewerkschaftlichen Kategorien nicht hinreichend erfassbar. Deshalb sucht der folgende Beitrag nach einem konzeptionellen Rahmen, der sich eignet, unterschiedliche Organisierungstypen im informellen Sektor zu erfassen und deren Ziele, die teils auf individuelle ökonomische Verbesserungen, teils auf strukturelle Veränderungen ausgerichtet sind, zu beleuchten. Entlang der Analyse unterschiedlicher Erwerbssegmente - Reproduktionsarbeit, Heimarbeit, Selbstbeschäftigung - und verschiedener Handlungsinitiativen und -strategien von informell arbeitenden Frauen sollen Kategorien abgeleitet werden, die eine konzeptionelle Einordnung von Selbstorganisierung ermöglichen. Vorauszuschicken ist den hier diskutierten Organisierungsformen, dass es sich um Suchprozesse von „New Voice“-Bewegungen ${ }^{1}$ handelt: Diejenigen verschaffen sich durch Selbstorganisierung eine Stimme, die bisher keine öffentliche Stimme hatten.

1 Der Begriff wurde in den USA angesichts des epidemischen Bedeutungsverlustes konventioneller Gewerkschaften geprägt. 


\section{Wie ,richtige“ ArbeiterInnen - Gewerkschaften von Hausangestellten}

In vielen Ländern des Südens stellen Hausangestellte die größte Berufsgruppe unter weiblichen Erwerbstätigen. In Namibia sind $46 \%$ aller berufstätigen Frauen Hausangestellte (Südwind 1994:73), in Brasilien ist ein Sechstel - in den Städten sogar ein Fünftel - aller weiblichen Erwerbstätigen in privaten Haushalten beschäftigt (1985 drei von 18 Millionen, Hosmer Martens/Mitter 1994:17). 80\% davon sind Schwarze.

Zugehfrauen, Kindermädchen und Putzhilfen agieren in einer Schnittfläche von bezahlter und unbezahlter Ökonomie jenseits formaler arbeitsrechtlicher Regelungen, indem sie Tätigkeiten aus der Sorge- und Haushaltsökonomie und der privaten Reproduktionssphäre als Erwerbsarbeit ausüben. Ihr Lohn wird häufig nicht vollständig monetarisiert, sondern teils in Naturalien ausgezahlt, teils mit Unterkunft und Verpflegung verrechnet. Weil viele Hausangestellte im Haushalt der Arbeitgeber wohnen, besteht einerseits eine starke persönliche Nähe zu den Arbeitgebern, andererseits ist für die erwerbstätigen Frauen eine Trennung von Privat- und Erwerbsleben äußerst schwierig (Schneider 1988; Eitel o.J.) Wie diese Arbeitsformen und Beschäftigungsverhältnisse eine Organisierung erschweren, soll exemplarisch am Beispiel der Hausangestelltengewerkschaft in Recife, Brasilien, gezeigt werden.

In Brasilien gab es bereits seit den fünfziger Jahren Organisierungsversuche von Hausangestellten. Anstöße zur Organisierung kamen aus kirchlichen Kreisen, von der Bewegung Junger Katholischer ArbeiterInnen. Diese stellten fest, daß sich Hausangestellte ihrer Tätigkeit schämten und sie angepasst an die allgemeine Wertung von Hausarbeit als nicht ökonomisch wertvolle und produktive Tätigkeit - nicht als Erwerbsarbeit definierten. Symbolische Demonstration, um die mangelnde Selbst- und Außenwahrnehmung als Arbeiterinnen zu überwinden, war der erstmalige Aufmarsch von Hausangestellten in der 1.-Mai-Parade 1963 in Recife.

Bei der Registrierung als Verein stießen die Haushaltshilfen und Kindermädchen zunächst auf bürokratische Hindernisse, weil sie keine Tätigkeitsnachweise hatten. Eine Registrierung als Gewerkschaft wurde erst möglich, nachdem die in mehreren Städten aktiven HausangestelltenVereine in der Neuformulierung der Verfassung von 1988 einige Arbeitsrechte für Hausangestellte und damit ihre Anerkennung als Berufstätige verankern konnten. Davor hatte die zuständige Verwaltung argumentiert: wo keine Berufstätigkeit, da keine Gewerkschaft.

In Recife wurde das Sindicato dos Trabalhadores Domesticos na Area Metropolitana da Cidade do Recife (STD) 1989 formal registriert. Auf dem Hintergrund des Strebens der Hausangestellten, als Arbeitnehmerinnen anerkannt zu werden, ist die Entscheidung des STD von 1992 zu sehen, sich 
einem der vier gewerkschaftlichen Dachverbände in Brasilien, der Zentralen Arbeiterunion CUT, anzuschließen. Obwohl die teils analphabetischen Frauen dort auf große Akzeptanzprobleme stoßen, wird die Integration als notwendig erachtet, um voll ,in die Welt der Arbeit“" einzutreten (Hosmer Martens/Mitter 1994:26). Inzwischen koordinieren die organisierten Hausangestellten ihre gewerkschaftliche Politik und ihre Forderungen nicht nur auf städtischer und nationaler, sondern auch auf kontinentaler Ebene im Verband Lateinamerikanischer und Karibischer Hausangestellter. Der Kampf um Akzeptanz und Rechte ist dabei der Schwerpunkt.

Zentrale Anliegen des STD sind bis heute die Aufwertung der Erwerbsarbeit der Hausangestellten und ihre formalrechtliche Gleichstellung mit anderen Berufsgruppen durch Anwendung von Arbeitsrechten, geregelten Arbeitszeiten und einem Beschäftigungsverbot von Kindern. Die Mitglieder werden für Verhandlungen und Auseinandersetzungen mit den ArbeitgeberInnen geschult, um sich den Verfügungsansprüchen der Patrona und der häufigen sexuellen Gewalt durch die Hausherren widersetzen zu lernen. Ein Hauptthema in den Beratungen des STD sind die Eintragungen in das Arbeitsbuch, in denen die Arbeitgeber den Beschäftigten Arbeitszeit, Löhne und gezahlte Sozialversicherungsbeiträge bestätigen. Mit diesem Nachweis können die Hausangestellten Anspruch auf Rente und Sozialleistungen erheben und im Konfliktfall rechtliche Schritte gegen die Arbeitgeber einleiten (vgl. Eitel o.J.: 33ff).

Rechtsberatung ist der Kern der Serviceleistungen des STD. Daneben bietet es seinen Mitgliedern Informationen und professionelle Rechtshilfe, Ausbildung, berufliche Weiterqualifizierung und Freizeitangebote. Organisierung eröffnet den Hausangestellten die Möglichkeit, ihre Isolation zu überwinden, aus der Unsichtbarkeit und Privatheit ihrer Arbeit herauszutreten, sich auszutauschen und ihr Selbstbewußtsein als Berufstätige und meist auch als Schwarze zu stärken, d.h. auf dieser Basis eine kollektive Identität herauszubilden. Problematisch ist, dass vor allem die jüngeren Mitglieder die Organisationen primär als Serviceinstitution sehen und schwer für ein Engagement zu gewinnen sind, weil sie hoffen, dass ihre Beschäftigung nur vorübergehend und Sprungbrett in höher gewertete und besser bezahlte Jobs sein wird. Wegen der Schwierigkeiten der Mobilisierung kommt der Öffentlichkeitsarbeit große Bedeutung zu.

Finanzierungsnöte sind chronisch beim STD, weil nur wenige Mitglieder es schaffen, die geforderten $2 \%$ ihres Lohn zu zahlen. Ein anderes Dauerproblem ist die beschränkte Reichweite und Mobilisierung der Gewerkschaft. Das STD ist sich des Defizits bewusst, dass es bisher weder Kinder in die Mobilisierung einbezieht noch Tagelöhnerinnen, die in Haushalten arbeiten, als ,selbstbeschäftigt“ gelten und deshalb nicht unter arbeitsrechtliche und Sozialversicherungsregelungen fallen. 
Außer dem Bündnis mit CUT pflegt das STD eine enge Zusammenarbeit mit der feministischen Gruppe SOS CORPO, die eine Stellvertreterfunktion in Bezug auf die Hausangestellten und ihre Interessen übernimmt. Ein zentrales Motiv für die Frauenorganisation, mit dem STD zu kooperieren, war das strategische emanzipatorische Interesse an einer Aufwertung von Reproduktionsarbeit - bezahlter wie unbezahlter - vor dem Hintergrund einer Infragestellung der geschlechtshierarchischen Arbeitsteilung. Zu Beginn der Koalition zwischen der feministischen und der gewerkschaftlichen Frauenorganisation gab es erhebliche Reibungen, die aus der Unterstellung einer kollektiven Geschlechtsidentität als unterdrückte Frauen von Seiten der mittelständischen Feministinnen in SOS CORPO erwuchsen. Diese Unterstellung einer vorrangigen Gemeinsamkeit teilten die Hausangestellten nicht, weil der Klassenunterschied zu den Mittelschichtsfrauen für sie im Vordergrund stand und eine Geschlechtsidentität überlagerte ${ }^{2}$. Für die Hausangestellten war (und ist) es ein Dilemma, dass sie in der Kooperation mit SOS CORPO und dem mittelschichtsorientierten Gewerkschaftsdachverband CUT immer wieder auf Vertreterinnen ihrer ArbeitgeberInnenklasse stießen. Trotz dieser Differenzen entstand eine fruchtbare Arbeitsallianz, und SOS CORPO unterstützt seit Jahren das STD bei seiner Lobby-, Bewußtseins- und Ausbildungsarbeit (Hosmer Martens/Mitter 1994: 27ff).

Um Haus- und Reproduktionsarbeit gesellschaftliche Anerkennung, Aufwertung und Rechtsansprüche zu verschaffen, ergriff in Mexiko eine kleine feministische Gruppe berufstätiger Mittelschichtsfrauen 1987 die Initiative zur Organisierung von Hausangestellten. Das feministische ATABAL Kollektiv und die Hausangestellten-Gruppe „La Esperanza“ agieren als Tandem: ATABAL fungiert als Beratungs- und Trainingsgruppe, „La Esperanza" kämpft für de jure und de facto Arbeitsrechte (ebd.: 42ff).

Anerkennungskämpfe waren auch der Dreh- und Angelpunkt der South African Domestic Workers Union (SADWU): Nach dreijährigen zähen Verhandlungen erkannte die Regierung 1993 offiziell den ArbeitnehmerInnenstatus von Hausangestellten an (Südwind 1994:69). ${ }^{3}$ Ebenso war für Hausangestellte in Namibia Anerkennung das Hauptmotiv, der Gewerkschaft beizutreten; sie war das Fundament für den Aufbau einer kollektiven Identität und die Voraussetzung für eine gewerkschaftliche Rechtspolitik. Mitglieder der Hausangestelltengewerkschaft waren die Frauen geworden, weil „,sie niemals als ,richtige“ Arbeiter wie im formellen Sektor betrachtet wor-

2 Die Geschichte der Hausangestelltengewerkschaft SINTRASEDOM in Kolumbien ist ähnlich verlaufen wie die des STD (Schneider 1988). Schneider beschreibt auch einen ähnlichen Konflikt zwischen Hausangestellten und Feministinnen in Bogota. Sie zog allerdings 1988 daraus den Schluss, dass in naher Zukunft ein Bündnis zwischen diesen Frauen nicht möglich sei.

3 Wenig später löste sich die SADWU aufgrund finanzieller Schwierigkeiten auf. 
den waren. Sie wollten mit demselben Respekt wie andere ArbeiterInnen behandelt werden und dieselben Zuschläge erhalten“" (Hosmer Martens/Mitter 1994:46).

Auch für eine andere Gruppe informell arbeitender Frauen in der Schnittfläche von bezahlter und Reproduktionsarbeit ist die Frage der Anerkennung als Lohnarbeiterin entscheidend für rechtliche und soziale Absicherung. Prostituiertenorganisationen kämpfen für die Legalisierung von Sexarbeit als Beruf, damit sie Ansprüche auf Sozialleistungen wie Gesundheitsversorgung und Rentenversicherung erheben können und ihre Arbeit rechtlichen Schutz, vor allem gegenüber der häufigen Gewalt, erhält. Die Prostituiertenbewegung in Brasilien, in Uruguay oder NGOs in Thailand wie „Empower“ gehen mit Aktionen und Kampagnen in die Öffentlichkeit und teils in die Gewerkschaftsbünde, um sich aus der Stigmatisierung und Unsichtbarkeit herauszubewegen und eine kollektive Berufsidentität zu schaffen (ila 1998: 4ff; ASW o.J.: 24ff). Gewalt, Gesundheit und Rechtsstatus der Sexarbeiterinnen sind ihre zentralen Themen, „Würde und das täglich Brot" sind - wie Rowbotham und Mitter allgemein für den informellen Sektor sagen - ihre Ziele. Als Reaktion auf die internationale Vernetzung von Prostituiertenorganisationen hat die ILO begonnen, sich mit dem Sex-Sektor als Berufsfeld, das arbeitsrechtlichen Regelungen zu unterwerfen ist, zu beschäftigen (Lim 1998).

\section{Marktfähigkeit steigern: Kooperativen und Kredite}

Die Beschäftigungsformen von Hausangestellten sind durch ein klares Arbeitgeber-Arbeitnehmer-Verhältnis konstituiert. Ihr Organisationsziel ist deshalb ein kollektives Aushandeln von Anerkennung, Arbeits- und Tarifrechten teils mit gewerkschaftlichen Methoden, teils mit Hilfe öffentlich erzeugten Drucks. Die Adressaten ihrer Forderungen - die Arbeitgeber sind eindeutig. Herkömmliche gewerkschaftliche Kampfformen laufen dagegen bei anderen Lohnarbeitsverhältnissen im informellen Sektor ins Leere. Als Heimarbeiterinnen in Indien für die Zahlung von Mindestlöhnen streikten, belieferten die Mittelsmänner sie nicht mehr mit Material, isolierten sie durch üble Nachrede und rekrutierten Heimarbeiterinnen in einer anderen Region. Gleichzeitig versuchten Arbeit- und Auftraggeber durch weitere Informalisierung die Verrechtlichung zu unterlaufen, indem sie die Heimarbeiterinnen in die „Selbständigkeit“ zwangen: Sie verkaufen die zu verarbeitenden Materialien an die Frauen und kaufen ihnen das Halbfertigoder Endprodukt dann wiederum ab. Damit entziehen sie sich z.B. in der Zigarettenindustrie der gesetzlichen Regelung, dass Mindestlohn und Arbeitsschutz auch für die knapp fünf Millionen Bidi-Arbeiterinnen gelten, die in Indien zu Hause Zigaretten rollen (Scheu 1995: 216ff). 
Weil die Arbeitsverhältnisse informell tätiger Frauen häufig zwischen Scheinselbständigkeit und Lohnabhängigkeit wechseln oder sich einer scharf abgegrenzten Definition entziehen, halten Hosmer Martens und Mitter (1996: 8) die Kombination von gewerkschaftlichen und genossenschaftlichen Ansätzen für eine effiziente Methode der Organisierung. Gewerkschaftliche Aktionsformen versuchen das Verhältnis zwischen den sehr unterschiedlichen Arbeit- bzw. Auftraggebern und den Beschäftigten bzw. Auftragnehmern im informellen Sektor zu regulieren, während Kooperativen die Isolation der (Schein-)Selbständigkeit überwinden, damit die Frauen als ökonomisches Kollektivsubjekt agieren können. Das kollektive Auftreten fördert die gesellschaftliche Anerkennung als ökonomische Akteurinnen, stärkt die kollektive Identitätsbildung und wirkt der Vereinzelung entgegen. Der Zusammenschluss ermöglicht die Ausschaltung von Mittelsmännern, stärkt die Verhandlungsposition in der verdichteten Konkurrenz des informellen Sektors und verbessert die Markt- und Verdienstchancen.

$\mathrm{Zu}$ Genossenschaften schließen sich meist Handwerkerinnen und Heimarbeiterinnen zusammen, die ihr Rohmaterial in großen Mengen erwerben, ihre Fähigkeiten und Produktionsmittel poolen und ihre Produkte gemeinsam vermarkten: Straßenhändlerinnen, Sammlerinnen von kleinen Waldprodukten, Bäuerinnen und Viehwirtinnen. Genossenschaftliche Organisierung kombiniert mit Qualifizierung und Management- und Vermarktungstraining stärkt die Verhandlungsmacht der Frauen und verbessert ihren $\mathrm{Zu}$ gang zu Markt und Produktionsmitteln.

Kooperativen verschreiben sich der ökonomischen Logik der Nutzenmaximierung. Dabei reintegrieren sie Kollektivität, Solidarität und Reziprozität in das ökonomische Handeln, ohne grundsätzlich den Wettbewerbsimperativ auf dem Markt in Frage zu stellen. Durch Rationalisierungsmaßnahmen und Qualifizierung verbessern sie die Marktchancen und erhöhen die Markteffizienz der kooperierenden Individuen.

Die meisten Genossenschaften haben jedoch große Probleme, profitabel zu arbeiten. Viele Produktionskooperativen von Frauen sind im Bereich des traditionellen Handwerks und der Kunstmanufaktur angesiedelt und einer starken Konkurrenz durch industrielle Produktion ausgesetzt. Die Beschaffung von Rohmaterialien ist oft schwierig und diskontinuierlich, weil die Industrie vorrangig und preisgünstiger bedient wird. Wo sie für den Export produzieren, werden neue Anforderungen an Materialien und Qualität gestellt, deren Erfüllung den Frauen häufig schwer fällt oder ihre Kosten erhöhen würde. Die Probleme mangelnder Wettbewerbsfähigkeit haben sich durch die Liberalisierung der neunziger Jahre noch weiter verschärft: der Druck auf Preise, Produktivität und Qualität wächst (Scheu 1995: 221). 
Neben genossenschaftlichen Strukturen gilt der Zugang zu Krediten im informellen Sektor als zentrales Vehikel zur Stärkung selbstbeschäftigter und scheinselbständiger Frauen, um sie von Verschuldung und aus der Abhängigkeit von lokalen Wucherern zu befreien und ihnen Zugang zu Investitionskapital zu verschaffen. Mikro-Kredite wurden in den letzten Jahren zunehmend als Zauberformel zur Armutsminderung und Marktintegration von Frauen gefeiert (Mayoux 1995: 33).

Dorf- und gemeindeorientierte Ansätze knüpfen mit ihren Mikro-Finanzierungsprogrammen häufig an informelle Spartöpfe von Frauen an, in die diese in Nachbarschafts- und Solidargruppen regelmäßig einzahlen. Chit funds in Indien, tontine in Westafrika, susu in Nigeria und merry-go-round in Ostafrika waren solch traditionelle Spargemeinschaften, in denen Frauen eine Rücklage für Sonderausgaben der Gemeinschaft im Katastrophenfall wie Missernte oder Dürre und für Rituale anlegten. Ein anderer Teil des Ersparten wurde und wird nach einem Rotationsprinzip als informelle Versicherung für den individuellen Notfall wie Krankheit oder für größere Anschaffungen oder Investitionen an die einzelnen Mitglieder ausgezahlt. Diese Gruppierungen waren konstitutiver Teil der moralischen Ökonomie, die primär auf dem Prinzip der Wechselseitigkeit beruhte, in der keine Trennung von Reproduktions- und ,produktiver“ Arbeit bestand und in der soziale Sicherheit unmittelbar aus der sozio-kulturellen Einbettung intergenerationeller und intergeschlechtlicher Verpflichtung erwuchs (Wichterich 1992, Schultz 1990). Mit dem Zusammenbruch solch reziproker Verpflichtungen durch die Erosion sozialer Bindungen gewinnen die informellen Solidargemeinschaften an Bedeutung, besonders für Witwen und alleinerziehende Mütter. Unter der Belastung der AIDS-Pandemie in Afrika haben viele dieser Gruppen ihren Fokus auf die Unterstützung bei Beerdigungen verlegt.

Kredit-Programme im informellen Sektor sind unterschiedlich organisiert. Häufig ist die Grameen-Bank in Bangladesh Vorbild für eine Mikro-Finanzierung (Fuglesang/Chandler 1988). Die Grameen-Bank vergibt Kleinkredite an inzwischen weit über zwei Millionen Frauen auf dem Land, vor allem als Startkapital für eine „einkommenschaffende Tätigkeit“. Die beiden Grundprinzipien sind, dass die Bank zu den armen Frauen geht, und nicht umgekehrt, und dass eine Gruppenhaftung eingeführt wurde, statt individuelle Sicherheiten zu verlangen. Die soziale Einbettung in die Gruppe und die moralische Verantwortung bewirken einen hohen Rückzahlungsdruck, der in die Literatur als hohe Rückzahlungsmoral von Frauen und als Erfolgsindikator für die Programme eingegangen ist (Mayoux 1995: 37). Die Grameen-Bank hatte ihre Mikro-Finanzierung zunächst Männern und Frauen angeboten. Als die Rückzahlungsquote bei Frauen erheblich besser war als bei Männern, konzentrierte sie sich auf Frauen als Kreditnehmerinnen. An- 
dere Organisationen in Asien und Afrika machten die gleiche Erfahrung mit der frauenspezifischen Schuldenbedienung (Heyzer 1994), allerdings findet das Prinzip der Gruppenbürgschaft in Afrika wenig Akzeptanz.

Hinter dem „Erfolg“ der Solvenz und zuverlässigen Rückzahlung durch die Frauen verbirgt sich allerdings häufig eine konsumtive Nutzung von Krediten statt der erhofften produktiven Investition, eine Weitergabe des Geldes an die Männer, die Aufnahme eines größeren Kredits bei einer anderen Organisation, um den ersten pünktlich zurückzahlen zu können. Relativ selten führt eine Kreditaufnahme zu erheblichen Einkommenssteigerungen (Mayoux 1995: 37ff, Khan 1998).

Die Grameen Bank bietet ihren Kundinnen auch Gesundheitsversorgung, Training und Zugang zu Produktionsmitteln für Einkommenserwerb an, vom Saatgut bis zu Mobiltelefonen, mit denen analphabetische Frauen in den Dörfern einen Telefondienst aufbauen können. Sie hat jedoch keinen gewerkschaftlichen oder genossenschaftlichen Ansatz, sondern verfolgt eher einen Service- und Fürsorgeansatz und organisiert die Frauen nicht als kollektives Handlungssubjekt.

Mikro-Finanzierungsprogramme mit Gruppenhaftung versuchen das Prinzip moralischer Verpflichtung in traditionellen Solidargruppen zu nutzen, doch sie torpedieren manchmal mit der Einführung des Geldprimats und der Verwertungs- und Rentabilitätsrationalität die soziale Einbettung dieser Gemeinschaften: Mehr Geld als in den herkömmlichen kleinen Spartöpfen wirkt offenbar als Anreiz, die Verantwortung zur Wechselseitigkeit zu ignorieren, und die Frauen entwickeln intern neue Dominanz- und Ausbeutungsstrukturen. Lediglich für einzelne in der Gruppe, keineswegs aber für alle, fungieren Kredite deshalb als Sprungbrett von den marginalisierten Rändern in zentrale Bereiche der Märkte (Mayoux 1995: 57).

Das 1978 in Südindien gegründete Working Women's Forum (WWF) baut dagegen zusätzlich zur Kreditvergabe auch Kooperativen auf und organisiert arme und informell arbeitende Frauen, mit dem Selbstverständnis, Teil der Frauenbewegung zu sein (Scheu 1995:195ff). Die Erfahrungen, die WWF mit der Vergabe von Krediten für Milchkühe machte, zeigen die Notwendigkeit eines multiplen, integrierten und kollektiven Ansatzes: Die hochgezüchteten Tiere starben, weil die Frauen individuell nicht in der Lage waren, das Futter und die Impfungen zu beschaffen. Kreditvergabe allein, ohne kollektive Unterstützungs- und Auffangmaßnahmen, ist also keineswegs ein Garant zur Existenzsicherung und erst recht nicht zur Verbesserung der Einkommenssituation (Heyzer 1994). Ebenso müsste die Haushaltsebene mit in die Bewusstseinsbildung und Mobilisierung einbezogen

4 Mitglied der Organisation wird eine Frau durch Beteiligung an dem Kredit- oder an dem Familienplanungsprogramm (letzteres verstanden als Methode der Armutsreduktion). 
werden, um zu verhindern, dass Frauen auf das Beschaffen von Darlehen für Männer reduziert oder nur als unbezahlte Zuarbeiterin von ihnen funktionalisiert werden (ebd.).

Das WWF fungierte zunächst als Mittlerin zwischen den Frauengruppen im informellen Sektor und dem formalen Finanzsektor und handelte mit diesem Sonderkonditionen aus. Inzwischen kann die Organisation einen großen Teil ihrer Kredite aus dem Kapitalstock ihrer Kooperativen vergeben, wodurch die Mitglieder die Kredite als „unser Geld“ betrachten. Dieses kollektive Besitzverhältnis findet sich noch stärker bei der Self-Employed Women's Association (SEWA) in Nordindien, wo die Mitglieder mit eigenen Spareinlagen bereits 1972 ihre ,eigene“ Bank als Kreditgenossenschaft gründeten.

Kredite wirken als Mobilisierungsvehikel im informellen Sektor, d.h. Frauen werden Mitglied einer Organisation, um einen Kredit zu bekommen (Carr/Chen/Jhabvala 1996:93). Die meisten Kredite werden jedoch - auch bei Gruppenbürgschaft - individuell genutzt. Lediglich kleine Frauengruppen in afrikanischen Dörfern verwenden das Geld als Kollektiv zur Anschaffung von Produktionsmitteln wie ein Gemeinschaftsfeld, einen Pflug, eine Mühle oder Vieh.

Kredite stärken die Marktfähigkeit der Frauen im informellen Sektor. Auch wenn sie nicht zu erheblichen Einkommenssteigerungen führen, werten sie die Frauen in der Familie und der lokalen Gemeinschaft als Subjekte auf, die Zugang zur Ressource Kapital haben, dienen der Absicherung ihrer Erwerbstätigkeit und der Stärkung oder Verstetigung ihrer Marktposition. Dabei bindet diese Mikro-Finanzierung grundsätzlich in die Marktrationalität ein und macht mit der Logik des Geld heckenden Geldes vertraut.

\section{Das „Modell“ SEWA}

Im Folgenden soll als Fallstudie die Selbst-Organisierung von Frauen im informellen Sektor unter dem Dach von SEWA nachgezeichnet werden, weil die Organisation typische Entstehungsbedingungen und Organisierungsanker, exemplarische Handlungsfelder und -strategien informell arbeitender Frauen aufweist, was aber nicht heißt, dass die SEWA-Strukturen und -Strategien leicht in andere Länder und Kulturen übertragbar sind oder gar ein Rezept darstellen, das überall zur Anwendung kommen sollte.

SEWA wurde 1972 von der Juristin und Gandhi-Anhängerin Ela Bhatt als Antwort auf ein institutionelles Defizit und das Versagen der konventionellen Textilarbeitergewerkschaft TLA im indischen Bundesstaat Gujarat gegründet. Bhatt, die über ein Jahrzehnt die Frauensektion der Gewerkschaft geleitet hatte, realisierte, dass die TLA weder in der Lage noch bereit war, die Problem- und Interessenlagen informell tätiger Frauen im Textilsektor - 
z.B. Lastenträgerinnen, die Stoffballen transportieren, Händlerinnen mit second-hand-Kleidung, Heimarbeiterinnen, die aus Fabrikabfällen Quilts nähen - in ihr gewerkschaftliches Aktivitätsspektrum aufzunehmen (Kabeer 1994: 240). Sie war spezifischen Frauenbelangen gegenüber ebenso indifferent wie dem informellen Sektor. Orientiert an einem patriarchalen, fordistisch inspirierten Ernährermodell bot die Frauensektion der TLA den Ehefrauen von industriellen Lohnarbeitern Näh-, Stick- und Tippkurse an, ignorierte jedoch die gleichzeitig stattfindende Ausgrenzung weiblicher Arbeitskräfte aus der Textilindustrie, die Deformalisierung ihrer Erwerbsarbeit und die Expansion des informellen Sektors. Die Beschäftigungsquote von Frauen in den Textilfabriken war zwischen 1950 und 1970 von 20\% auf weniger als 3\% gesunken (Sebstadt 1982: 234). Die TLA blendete die informellen $\mathrm{Zu}$ - und Weiterverarbeitungssegmente auf den urbanen Märkten und im Hinterland, die sich wie Ringe um die formale Produktion legen, schlichtweg aus.

Die Registrierung von SEWA als Gewerkschaft stieß zunächst auf bürokratischen Widerspruch des Arbeitsministeriums, das argumentierte: Wo kein klar konturierter Arbeitgeber, da ist auch kein Bedarf für eine Gewerkschaft. SEWA konterte, dass die oberste Gewerkschaftsaufgabe die Verteidigung von ArbeiterInneninteressen sei, auch wenn der Arbeitgeber nicht klar identifizierbar sei (Kabeer 1994: 232).

Als Gewerkschaft registriert, nahm SEWA immer mehr Berufsgruppen und Erwerbsfelder des informellen Sektors unter ihr selbstdefiniertes Dach der „Selbstbeschäftigung“. Der Begriff wurde bewußt gegen Negativ- und Defizitdefinitionen des informellen Sektors gesetzt und umfaßt im Verständnis von SEWA folgende drei Kategorien erwerbstätiger Frauen: 1) Kleinhändlerinnen, 2) Heimarbeiterinnen, die im Familienbetrieb oder auf Stücklohn arbeiten, 3) Tagelöhnerinnen sowie Teilzeit- und Saisonarbeiterinnen im Dienstleistungsbereich, der Landwirtschaft und auf dem Bau (SEWA 1997: 4).

Im Kontext blutiger Kastenauseinandersetzungen in Gujarat kam es 1981 zur Abtrennung der SEWA von der Vatergewerkschaft TLA ${ }^{5}$. Der Konflikt schien der TLA ein willkommener Anlass zu sein, um die eigenen Lohnarbeiter-Partikularinteressen durch den Ausschluss der abtrünnigen Tochter, deren Mitgliedschaft rasch wuchs und die national und international zunehmend Aufmerksamkeit erregte, klar abzugrenzen (Sebstad 1982: 219ff, Rose 1992: 74ff). SEWAs „Entwicklungsaktivitäten“ wie die Vergabe von Krediten und der Aufbau von Kooperativen, waren der TLA ein Dorn im

5 SEWA ergriff im Unterschied zur TLA Partei für die Dalits und gegen höherkastige Hindus und trat für eine Politik positiver Diskriminierung und Quotierung zugunsten von Dalits ein, auch weil viele SEWA-Mitglieder Dalits oder niedrigkastig sind. Daraufhin wurden Bhatt und SEWA aus der TLA ausgeschlossen. 
Auge, weil sie angeblich die Verfolgung gewerkschaftlicher Taktiken behinderten. Die Separierung von der Vater-Organisation markiert die Geburt von SEWA als autonomer Frauengewerkschaft, die intensivierte Suche nach neuen Organisationsformen und Handlungsansätzen und die Entscheidung zur Ausdehnung in ländliche Regionen. Sie schlug einen geschlechtsseparatistischen Weg unabhängig von der männlichen Gewerkschaftsbewegung ein, weil es für innovative und über gewerkschaftlichen Aktivismus hinausgehende Maßnahmen unter ihrem Dach nicht genug Spielraum gab. Heute hat SEWA in Gujarat doppelt so viele Mitglieder auf dem Land wie in den Städten: insgesamt 220 000, davon über 100000 in den ländlichen Regionen Gujarats, 56000 in den Städten des nordindischen Bundesstaats, und der Rest in fünf anderen Bundesstaaten.

\section{Strategien}

Ausgangspunkt für SEWA waren gewerkschaftliche Methoden, die sie an ihre heterogene Klientel im informellen Sektor anpasste, und nach und nach um entwicklungsorientierte und frauenpolitische Maßnahmen erweiterte. So entstand ein Paket von Programmen, Aktivitäten und Taktiken, das zu erheblichen Teilen durch Mittel ausländischer Geber finanziert ist. Bildhaft stellt SEWA sich heute als Banyan-Baum, der das Versammlungszentrum und der Gemeinschaftsort in vielen indischen Dörfern ist, mit vielen Luftwurzeln und sieben tragenden Ästen dar: Gewerkschaft, Bank, ländliche Entwicklung, soziale Sicherheit, Kooperativen, Akademie und politischer Wandel. Bereits in dem Konflikt mit der TLA erklärte Bhatt die Kombination der unterschiedlichen Ansätze zur Strategie:

„Druck und Entwicklung oder Gewerkschaft und Kooperative - durch die Verknüpfung der beiden, durch beide Arme war SEWA in der Lage, die Arbeiterinnen aus Ausbeutung und Erwerbslosigkeit zu befreien." (zit. in: Gandhi/Shah 1992: 282).

Ausgehend von dem Kernziel ökonomischer Besserstellung wurden die Handlungsziele sukzessive ausgedehnt. Ihre Aktivitäten und Ziele leitete die Organisation aus Bedarfsanalysen verschiedener Segmente des informellen Sektors und aus den Problemen, wie sie von den informell arbeitenden Frauen identifiziert wurden, ab. Dann ließ sie die Frauen selbst die Handlungsprioritäten bestimmen, Maßnahmen konzipieren und - nach einem entsprechenden Training - Initiativen durchführen. Diese Vorgehensweise ist partizipativen Forschungsansätzen der entwicklungsorientierten und armutsbekämpfenden Basis- und Gemeindearbeit von NGOs entlehnt und löste eine Kettenreaktion von Aktivitäten und Programmen aus. Die Vergabe von Krediten wurde als Reaktion auf die hohe Verschuldung bei lokalen Wucherern beschlossen; als Ursache für die schlechte Rückzahlung wurden Gesundheitsprobleme ausgemacht, was zur Folge hatte, dass eine 
Gesundheitsversorgung für die Frauen unter Beteiligung von entsprechend ausgebildeten SEWA-Mitgliedern als „barefoot-doctors“ aufgebaut wurde; schließlich wurden von den Frauen selbst gemanagte Kinderhorte als Bedingung für Produktivitätssteigerung und die Ausdehnung der Arbeitszeit eingeführt usw.

Jede der SEWA-Vereinigungen in den sechs Bundesstaaten wendet die partizipativen Methoden der Problemidentifikation und Planung an und leitet daraus kontextabhängig unterschiedliche Organisierungs- und Aktionsschwerpunkte ab. Das Prinzip der Integration von ökonomischen, sozialen und frauenpolitischen Maßnahmen ist übertragbar, seine konkrete inhaltliche und strategische Ausfüllung jedoch jeweils von den spezifischen Arbeits- und Problemsituationen der Frauen, ihren kulturell umrissenen Handlungsrahmen und den politischen Rahmenbedingungen abhängig.

Weil nicht nur Bedürfnisidentifikation und Planung, sondern auch die Durchführung von Programmen von den Mitgliedern an der Basis selbst getragen und in demokratische Entscheidungsstrukturen eingebettet werden, bestand von Anfang an eine hohe Identifikation, ja ein Besitzgefühl der Mitglieder gegenüber ,ihrer“ Organisation. Dazu gehört auch, dass die mittelständischen Frauen in Leitungspositionen - vor allem die charismatische Gründungs- und Führungsgestalt Bhatt - ihre Klassen- und Kastenlage reflektieren, demokratische Strukturen aufbauen und bemüht sind, Basisfrauen Management und Führung der Organisationen zu übertragen (Rose 1992). SEWA versteht sich selbst als ,beides, eine Organisation und eine Bewegung“ und verortet sich innerhalb der sozialen Bewegungen Indiens.

„SEWA als Bewegung ist gestärkt dadurch, dass in ihr drei Bewegungen zusammenfließen: die ArbeiterInnenbewegung, die Kooperativen-Bewegung und die Frauenbewegung. Es ist eine Bewegung selbstbeschäftigter Arbeiterinnen, ihre eigene, hausgemachte Bewegung mit Frauen als Führerinnen. Durch ihre eigene Bewegung werden Frauen stark und sichtbar. Durch ihren Glauben an Organisierung für die eigenen Rechte, zum Schutz des Überlebens (livelihood) und Sicherung ihres rechtmäßigen Platzes in der Ökonomie haben sich selbstbeschäftigte Frauen positioniert. Jetzt sind sie sichtbar, sind artikulierter und selbstbewußter." (SEWA 1997: 3)

Mit dieser emphatischen Selbstdarstellung als Bewegung betont SEWA ihre Basisverankerung, den Prozesscharakter der Selbstorganisierung und den „Besitz“ der Mitglieder an der Organisation, was eine Abgrenzung gegen ein Verständnis als Dienstleistungs- und Wohlfahrtsinstitution impliziert.

SEWA hat eine kollektive Identität ihrer Mitglieder aufgebaut, die sich aus ihren sozial konstruierten Identitäten als Frauen, als Kastenangehörige und als Selbstbeschäftigte zusammensetzt. Die positive Besetzung und Aufwertung der ökonomischen Tätigkeit im informellen Sektor ist Grundlage eines neuen Selbstbewußtseins, dass die Frauen nach innen, für sich selbst, und nach außen, gegenüber der formalen Ökonomie und der städtischen Öffent- 
lichkeit gewinnen. Anerkennungspolitik versteht SEWA als Politik der Sichtbarmachung.

Durch ihre Aktionen und Kämpfe bilden die Frauen einerseits ,power from within“ (Kabeer 1994: 245ff), durchbrechen andererseits ihre soziale Ausgrenzung und machen sich im öffentlich-politischen Raum sichtbar und hörbar (Chen 1996: 50). Sie setzen sich als kollektives Handlungssubjekt, als politische und ökonomische Akteurinnen, indem sie ihre Interessen artikulieren lernen und sich Handlungsraum erobern ${ }^{6}$. So erstritt SEWA mit hoher Öffentlichkeitswirkung per Gericht von der Stadtverwaltung in Ahmedabad, dass Kleinhändlerinnen ein Raum auf den großen städtischen Märkten zugesichert wurde, der nach der formellen Übergabe dann noch von Gewalt und Bestechungszwang freigekämpft wurde. Der Kampf um einen legitimierten Raum auf den Märkten ist - neben den Auseinandersetzungen um die Preise, die sie Großhändlern, die häufig auch als Geldverleiher fungieren, für die Waren zu zahlen haben - ein Teil des ZweiFronten-Kampfes, den Straßenhändlerinnen überall zu führen haben. In Südafrika verfolgt die Self-Employed Women's Union (SEWU) nach dem SEWA-Modell die gleiche Handlungsstrategie (Afrika Süd Aktionsbündnis 1996, Horn 1997), in Ruanda eine Vereinigung von Händlerinnen mit second-hand-Schuhen (Chirangashane 1998).

Die Markierung und Legitimierung des ökonomischen Orts der Straßenhändlerinnen war maßgeblich für SEWAs Profilierung als Instanz kollektiver Interessenvertretung und für die Sichtbarkeit der Frauen. Auch die SEWA-Akademie als Bewußtseinsbildungs- und Trainingsinstitution und die Video-Einheit, in der Mitglieder SEWA-Aktivitäten zur Schulung, Mobilisierung und für die Öffentlichkeit dokumentieren, tragen gezielt zur Selbstvergewisserung der kollektiven Identität und der Herausbildung von Selbstvertrauen und Außendarstellung bei.

Der Zugewinn an Selbstbewußtsein und die öffentliche Sichtbarkeit sind das Fundament für eine stärkere Verhandlungsmacht. Neue Formen kollektiver Verhandlungsmacht entwickelten die SEWA-Frauen in gewerkschaftlichen Kämpfen für Mindestlohn, für Verkaufslizenzen für Straßenhändlerinnen und die Anwendung der Arbeitsschutzgesetzgebung auf den informellen Sektor. Durch öffentliche Aktionen wie Demonstrationen, Marktplatz- und Behördenbesetzungen und Vorgaben für die Stadtplanung, durch Lobbyarbeit, Memoranden und Resolutionen an die Regierung des Bundesstaates und die nationale Planungskommission profilierte die Organisation sich als ein Intermediär zwischen den Interessen informell tätiger Frauen und den politischen Institutionen der Städte und des Staates, d.h. sie trat als

6 Die beiden Kategorien „voice“ und „space“ tauchen in Empowerment-Konzepten für Frauen immer wieder als Standardbausteine auf (Batliwala 1993, Kabeer 1994). 
zivilgesellschaftlicher Stakeholder auf. In ihrer Lobbyarbeit als Pressure Group betreibt SEWA einen Drei-Ebenen-Ansatz im lokalen Politikgeschehen, in der nationalen und in der internationalen Arena, z.B. für die 1996 verabschiedete ILO-Konvention zur Heimarbeit.

Zwar übt die Organisation unmissverständlich Kritik an unzureichenden gesetzlichen Regelungen und ihrer mangelhaften Umsetzung, an behördlichen Planungen und Programmen, die blind gegenüber Frauen und dem informellen Sektor sind, und an der Korruption des Verwaltungsapparats. Trotzdem kooperiert SEWA aus einer staatsaffirmativen Grundhaltung heraus mit städtischen Administrationen und Behörden, nutzt Regierungsprogramme oder bietet sich als Implementierungs- und Optimierungsinstanz an, versucht durch Öffentlichkeitsarbeit, Lobbying und konstruktive Politikvorlagen Einfluss zu nehmen. Wo institutionalisierte Politik, staatliche Programme und Gesetze unzureichend sind, hat SEWA alternative Institutionen aufgebaut, um Defizite im Bereich Gesundheitsversorgung, der sozialen Sicherung, der Kinderbetreuung, des Wohnungsbaus, der Trinkwasserversorgung und Wiederaufforstung zu kompensieren.

Strategisch arbeitet SEWA auf eine Verrechtlichung und Formalisierung des informellen Sektors hin. Nach jahrelangem in die Öffentlichkeit getragenen Kampf und gerichtlichen Klagen konnte sie z.B. durchsetzen, dass die scheinselbständigen Bidi-Heimarbeiterinnen unter die Arbeitschutzgesetzgebung fallen (Scheu 1995:218). Gegen die fortgesetzte Unterlaufung und Missachtung von rechtlichen Regulierungen innerhalb der informellen Arbeitsverhältnisse ist sie jedoch immer wieder machtlos. Die Umsetzung der Gesetze bleibt vom Willen oder eben der Willkür der Auftraggeber und Mittelsmänner abhängig.

Um Abhängigkeiten zu reduzieren und Zugang zu Ressourcen zu eröffnen, vergibt SEWA zum einen Kredite ${ }^{7}$. Zum anderen fördert sie - um die Isolation von Arbeiterinnen in den Überlebensökonomien zu überwinden - $\mathrm{Ge}$ nossenschaften in mehr als 80 verschiedenen Bereichen, mit unterschiedlichen Erfahrungen. Es zeigte sich, dass durch die Kooperativen zwar die Marktfähigkeit und -einbindung der Frauen verbessert werden konnten, Absatz- und Verdienstsicherheiten konnte SEWA jedoch nicht schaffen (Scheu 1995:221, Rowbotham/Mitter 1994: 221). ${ }^{8}$

Angereichert wird dieses Paket von ökonomischen und sozialen Sicherungsprogrammen um frauenpolitische und emanzipatorische Maßnahmen.

7 Die Mitglieder einer Berufsgruppe müssen der Darlehensvergabe an eine Frau zustimmen. Es besteht jedoch kein Gruppenhaftungssystem.

8 Es kam auch zu Konflikten mit Genossenschaftsmitgliedern, die SEWA in der Rolle eines Arbeitgebers sahen, weil sie z.B. die Kooperative der Stoffdruckerinnen und Papiersammlerinnen managt und den Frauen einen Lohn auszahlt. Diese forderten von SEWA einen festen Monatslohn, obwohl die Kooperativen Verluste machten. 
Wesentlich ist, dass immer wieder unbezahlte Reproduktions- und Sorgearbeit von Frauen (z.B. Kinderbetreuung und Krankenpflege, Versorgung mit Trinkwasser, Brennenergie und Futter für das Vieh) in das Aktivitätsspektrum von SEWA einbezogen wird. Dazu gehören auch Versuche, die geschlechtsspezifische Arbeitsteilung aufzuweichen.

Nicht nur hat SEWA die alte Forderung der Arbeiterinnenbewegung nach gleichem Lohn für gleiche Arbeit z.B. beim Straßenbau durchgesetzt. Sie bildet auch Frauen in „männertypischen“ Berufsfeldern aus, um die geschlechtshierarchische Segmentierung des Arbeitsmarkts zu durchbrechen, und motiviert ihre Mitglieder, mit ihren Männern die geschlechtsspezifische Arbeitsteilung im Haushalt auf der Grundlage neu auszuhandeln, dass die Wertschätzung der Frauen innerhalb ihrer Familien mit dem Einkommen, den Ressourcen und Gütern, die sie in den Haushalt einbringen, wächst. Einige Ehemänner übernehmen inzwischen Haus- und Sorgearbeit, um ihre Frauen für den Einkommenserwerb zu entlasten (Carr/Chen/Jhabvala 1996: 99f, 122f). Häufig stoßen die Frauen jedoch auf Widerstände, bei den eigenen Männern oder in der Gemeinschaft, die sie nicht in Männerberufen akzeptiert. In der Kaste der Aahir verbot ein Ältestenrat als Reaktion auf die erweiterten Handlungsräume den Frauen sogar, allein das Dorf zu verlassen und an Trainingskursen teilzunehmen (ebd.: 123ff).

SEWA kooperiert mit Frauenorganisationen, versteht sich als aktiven Teil der sehr vitalen und vielfältigen, indischen Frauenbewegung und hat sich in deren Kampagnen gegen Gewalt in der Familie, Mitgift und vorgeburtliche Geschlechtsbestimmung eingeklinkt. Wie in Bezug auf die informelle Erwerbsarbeit verfolgt sie auch bezüglich frauendiskrimierender Strukturen zu allererst eine Politik der Thematisierung und Sichtbarmachung.

\section{Macht zum Handeln}

Typisch für die Organisationen informell arbeitender Frauen ist die strategische Kombination unterschiedlicher Initiativen, Methoden und Ziele. Konzeptionell lässt sich diese Integration ökonomischer, sozialer und frauenpolitischer Ansätze unter dem Begriff des Empowerment subsummieren (vgl. Carr/Chen/Jhabvala 19969 ${ }^{9}$, Batliwala 1993, Kabeer 1994, Scheu 1995). In Anknüpfung an Hannah Arendt wird Macht positiv verstanden als Fähigkeit, Energie und strukturelle Möglichkeit zu handeln und zu verhandeln, Widerstand gegen Herrschaft zu leisten, zu definieren und zu entscheiden, zu gestalten und auszugleichen. Macht ist Kraft zur Veränderung oder - wie Kate Young sagt (1993: 157) - Transformationspotential.

9 Carr/Chen/Jhabvala unterscheiden vier verschiedene Ansätze zum ökonomischen Empowerment: Dorf- und Gemeinschaftsorientierung, Kooperativen, Banken, Gewerkschaften. 
Empowerment-Konzepte für Frauen verknüpfen deren praktische Bedürfnisse mit ihren strategischen Interessen an einer Veränderung der hierarchischen Geschlechterbeziehungen. Sie basieren auf Organisierung als wesentlicher Strategie kollektiver Machtbildung und zielen auf den Umbau ungleicher Machtstrukturen zugunsten von Frauen. Inspiriert wurden die Machtbildungsstrategien für Frauen durch die US-amerikanische BlackPower-Bewegung der sechziger Jahre, durch Saul Alinskys Anleitung zum Mächtigsein aus der Arbeiter- und Bürgerrechtsbewegung der USA und durch Paolo Freires Pädagogik der Unterdrückten. Batliwala definiert Empowerment als

„Prozess, in dem die Machtlosen oder Entmachteten mehr Kontrolle über Ressourcen und über Entscheidungen gewinnen... Die Ressourcen, über die Kontrolle hergestellt wird, fallen in fünf Kategorien: physische Ressourcen (wie Land, Wasser, Wald); menschliche Ressourcen (Menschen, ihre Körper, ihre Arbeitskraft und Fähigkeiten); intellektuelle Ressourcen (Wissen, Information, Ideen); finanzielle Ressourcen (Geld, Zugang dazu) und das Selbst (diese einzigartige Kombination von Intelligenz, Kreativität, Selbst-Bewußtsein und Vertrauen) (1993: 3, 7).

Entsprechend nennt Batliwala als Indikatoren für ökonomisches Empowerment nicht nur ein wachsendes Einkommen, sondern auch verbesserte Gesundheit und Ernährung, ein größeres Selbstbewußtsein als ökonomische Akteurin, mehr Verhandlungsmacht in der Familie und auf dem Markt und Zugang zu Ressourcen wie Kredit (ebd.: 36ff).

Mayoux unterscheidet bei ökonomischen Ansätzen für Selbstbeschäftigte

„einen Markt-Ansatz, der darauf abzielt, einzelnen Kleinunternehmerinnen zu helfen, ihr Einkommen zu erhöhen ${ }^{10}$, und einen Empowerment-Ansatz, der nicht nur die Einkünfte erhöhen will, sondern auch die Verhandlungsmacht armer Produzentinnen durch Gruppenaktivitäten steigern will.“( 1995:4)

Hier werden Übereinstimmungen und Schnittflächen zwischen dem Empowerment-Konzept und den Entitlement- und Well-being-Ansätzen ersichtlich, die Amartya Sen aus seiner Forschung zu Armut und Hunger, Haushalt und Autonomie abgeleitet hat (Sen 1982, 1990).

Frauenorganisationen im informellen Sektor öffnen ihren Mitgliedern Zugänge zu Rechten, zum Markt und zu Ressourcen wie Kredit und Produktionsmitteln, Land und Wald, Training, Gesundheit und sozialer Sicherheit. Diese Öffnung von Zugangsmöglichkeiten, Nutzungs- und Verfügungsrechten im Öffentlichen und Privaten ist ein Ermöglichungsprozess, der sich mit dem von Sen entwickelten Entitlement-Ansatz fassen lässt. Um den ökonomischen und armutsbekämpfenden Kern haben die Organisationen dabei (unterschiedlich) komplexe Aktionsradien mit dem Ziel des Wohlergehens (well-being) informell arbeitender Frauen entwickelt.

10 Diesen sieht sie exemplarisch in Programmen der Weltbank verfolgt. Er entspricht den Ansätzen, die Caroline Moser ,effizienz-orientiert“ genannt hat. 
Das Empowerment-Konzept ist inzwischen von Regierungen und UN zur Alleskleber-Formel geschliffen worden, wird dort aber häufig als Steuerungs- oder Wohlfahrtsprogramm von oben verstanden. Daraus ergibt sich die Frage: wer empowert wen mit welchem Ziel? Im Gegensatz zu staatlichen Top-Down-Strategien begeben sich die Frauenorganisationen in einen Prozess des Self-Empowerment (DAWN 1985), erkämpfen sich in kleinen Vor- und Rückschritten Einflussmöglichkeiten, Rechte und Ressourcen, die die Frauen selbst identifizieren und in Strategien zu strukturellen Veränderungen einbauen. Die Konstitution eines kollektiven Handlungssubjekts ist eine Form selbstbestimmter Vergesellschaftung.

Charakteristisch für diese Selbstorganisierung ist, dass über die marktförmige Existenzsicherung hinausgegangen wird, d.h. dass die ökonomischen Maßnahmen in soziale integriert werden, dass sie mit Gesundheitsversorgung, Notfall- und Alterssicherung sowie Kinderbetreuung und frauenemanzipatorischen Ansätzen verbunden werden. SEWA zielt dabei am umfassendsten auf eine Verbesserung der gesamten Lebensbedingungen armer, informell arbeitender Frauen und auf strukturelle gesellschaftliche und politische Transformationen in ihrem Umfeld. Weil nur eine kleine Gruppe der SEWA-Mitglieder in einem direkten oder dauerhaften Beschäftigungsund Lohnarbeitsverhältnis steht, sind entwicklungsorientierte Ansätze für sie strategisch erfolgversprechender als gewerkschaftliche. Hausangestellte, die in einem klaren Arbeitgeber-Arbeitnehmerverhältnis stehen, verfolgen dagegen primär gewerkschaftliche Strategien mit dem Kern der Anerkennungspolitik. Frauenspezifisch ist dabei in jedem der Berufsfelder die Mobilisierung von Gegenwehr gegen sexualisierte Gewalt.

Indem die Organisationen Frauen als ökonomische Akteurinnen stärken, wirken sie strategisch dagegen, dass die Ungleichheitsstrukturen des Marktes auf der vorgeordneten Geschlechterungleichheit aufbauen und diese gleichzeitig nutzen und reproduzieren. Der frauenemanzipatorische Ansatz ist bei den Organisationen am stärksten, die zum einen Probleme der marktförmigen Erwerbsarbeit und der unbezahlten Sorgearbeit integriert bearbeiten und zum anderen die Entscheidungs- und Verteilungsstrukturen sowie die „kooperativen Konflikte“ (Sen 1990) innerhalb des Haushalts mit in den Blick nehmen. Sie verfolgen somit nicht nur die strategische Perspektive einer Aufwertung von (unbezahlter wie bezahlter) Reproduktionsarbeit, sondern auch des Aufbrechens der geschlechtshierarischen Arbeitsteilung auf den Erwerbsmärkten und in den Haushalten.

Allgemein gilt, dass ökonomisches Empowerment von Frauen auf dem Markt einer Absicherung in der Privatsphäre bedarf. Denn verbesserte Marktchancen, ein sicheres Einkommen und Zugang zu Ressourcen führen nicht zwangsläufig zu mehr Entscheidungs- und Verfügungsmacht von Frauen über Ressourcen und Einkommen innerhalb des Haushalts. In den 
verschiedenen real-existierenden patriarchalen Haushaltsstrukturen besteht häufig die Gefahr, dass Männer sich umgehend das Einkommen von Frauen aneignen oder auch zur Legitimierung ihres Rückzugs aus der Ernährungsverantwortung missbrauchen. Dagegen gibt ein eigenes Einkommen Witwen, alleinerziehenden und geschiedenen Frauen die Möglichkeit, unabhängig von männlichen Verwandten zu leben.

Nicht einer der Ansätze allein, sondern die Kombination mehrerer Ansätze und ein integriertes Vorgehen vermögen, den Frauen Anerkennung nach innen wie nach außen und Anspruchsrechte zu bringen. Die häufige Unsichtbarkeit informeller Arbeit wird durchbrochen, die Wettbewerbsfähigkeit der Frauen durch Qualifizierung und Zugang zu Produktionsmitteln verbessert. Dies mag die informell Arbeitenden absichern und Risikominimierung bewirken, weitere Marginalisierung und extreme Auswüchse von Ausbeutung und Rechtsverweigerung verhindern, trotzdem bleibt es schwierig, eine nachhaltige Existenzsicherung aufzubauen oder gar gegen die Tendenz zu weiterer Informalisierung, Liberalisierung und Verdichtung von Konkurrenz nennenswerte Einkommensverbesserungen durchzusetzen.

Durch Bildung kollektiver Handlungsmacht und Identität sowie durch Maßnahmen sozialer Sicherung versuchen die Frauenorganisationen, Elemente der moralischen Ökonomie in den Binnenräumen der Gruppen zu reinstallieren oder tradierte Strukturen, die dies leisten, zu erhalten. Kooperation und Reziprozität sind aus der Not geboren, aber die Organisationen sind weit mehr als Notgemeinschaften. Kooperation und Solidarität sind Gegenwerte und Gegenstrukturen zur individualisierenden Konkurrenzlogik des Marktes. Herausragendes Beispiel dafür waren die Versuche von SEWU in Südafrika, migrierende Händlerinnen als Mitglieder zu gewinnen, die Waren aus Nachbarländern über die Grenzen schmuggeln und versuchen, lokale Händlerinnen zu unterbieten (SEWU 1999).

Damit sind die Gruppen bemüht, das mikro-ökonomische Handeln in soziale Zusammenhänge einzubetten, begeben sich aber nicht in grundsätzliche Opposition zu marktökonomischen Imperativen und Kalkülen. Vielmehr rüsten sie ihre Mitglieder mit Qualifikationen und Ressourcen für die marktwirtschaftliche Konkurrenz. Bei individuell genutzten Krediten - und dies sind die meisten auch beim Prinzip der Gruppenhaftung - besteht jedoch die Gefahr, dass sie eine Ausbettung aus dem Sozialen und ein Aufbrechen der moralischen Zusammenhänge auslösen.

Aufgrund der Schwierigkeiten, sich selbst zu finanzieren, sind die Frauenorganisationen im informellen Sektor stark abhängig von ausländischen Mitteln und deshalb organisatorische und institutionelle Strukturen ohne Bestandsgarantie. Ihre entscheidende Leistung besteht darin, ökonomische, soziale und frauenrechtliche Absicherungspolitik zu betreiben und die „Verletzbarkeit“" (vulnerability) von informell tätigen Frauen durch kollek- 
tive Identitäts- und Machtbildung zu reduzieren. Sie schaffen Sicherungsstrukturen, auf die die Mitglieder zurückgreifen können, wo die tradierten familialen Auffangstrukturen zunehmend erodieren.

In den Kategorien von Empowerment-Konzepten gesprochen bauen Frauenorganisationen im informellen Sektor Verhandlungsmacht der informell tätigen Frauen auf, verhelfen ihnen zu Verfügungsmacht über Rechte und Ressourcen und bemühen sich um Gestaltungsmacht in der Politik und Ökonomie. Sie bilden eine kollektive Identität der Selbstbeschäftigten als Handlungsgrundlage, führen Aktivitäten in strategischer Vielfalt durch und bauen organisatorische Macht um die Säulen von Anerkennung, Rechten und Ressourcen auf. Dabei stellen sie eine neue Form der Vergesellschaftung dar und kämpfen gegen die Vergeschlechtlichung von Arbeit und der Märkte durch die geschlechtshierarchische Arbeitsteilung und die entsprechende Segmentierung des Marktes, die Frauen die schlecht bezahlten, niedrig bewerteten Arbeiten zuweisen. Sie betreiben Ermöglichungs- und Ermächtigungspolitik - mit Erfolgen, Brüchen und Rückschlägen. Letztlich bricht sich das ökonomische Empowerment der Frauen jedoch immer wieder an dem strukturellen Widerspruch, dem die Formalisierungsversuche des informellen Sektors unterliegen.

\section{Literatur}

Afrika Süd Aktions-Bündnis (1996): Die südafrikanische Frauengewerkschaft SEWA, Bonn.

Batliwala, Srilatha (1993): Education for Women's Empowerment. A Conceptual Analysis. Mimeo, FAO/ASPBAE, Bangalore.

Carr, Marilyn; Chen, Martha; Jhabvala, Renana (1996): Speaking out. Women's Economic Empowerment in South Asia, New Delhi.

Chen, Martha Alter (1996): Beyond Credit, A Subsector Approach to Promoting Women's Enterprises, Ottawa.

Chirangashane, Louise Lubala (1998): Das Verkaufen von gebrauchten Schuhen - das Ringen um Überleben im Süd-Kivu, in: Frauen in der einen Welt, 2/1998, S.116-126.

DAWN, (1985): Development, Crises, and Alternative Visions: Third World Women's Perspectives, Stavanger.

Eitel, Christiane (o.J.): Der Kampf der brasilianischen Hausangestellten - die Hausangestelltengewerkschaft von Recife, in: ASW, Traumwelt, Migration und Arbeit, S. 31-37.

Fuglesang, Andreas; Chandler, Dale (1988): Participation as process - what we can learn from Grameen Bank, Bangladesh.

Heyzer, Noeleen (1994): Increasing Women's Access to Credit in Asia: Achievements and Limitations, in: Heyzer, N.; Sen, G. (eds.), Gender, Economic Growth and Poverty, New Delhi.

Horn, Pat (1997): A labour policy for all, in: Agenda, No 35, S.51-55.

Hosmer Martens; Mitter, Swasti (eds.) (1994): Women in Trade Unions. Organizing the Unorganized, Geneva.

ila (1998): ila 213 Prostitution.

Kabeer, Naila (1994): Reversed Realities. Gender Hierarchies in Development Thought, New Delhi.

Khan Osmani, Lutfun (1998): The Grameen Bank Experiment: Empowerment of Women through Credit, in: Afshar, Haleh (Ed.): Women and Empowerment, New York, 67-87.

Lim, Lean Lin (1998): The Sex Sector. The economic and social bases of prostitution in Southeast Asia, Geneva. 
Mayoux, Linda (1995): From Vicious to Virtous Circle? Gender and Micro-Enterprise Development, Geneva.

Razavi, Shahra (1998): Gendered Poverty and Social Change, UNRISD, Geneva.

Rose, Kalima (1992): Where Women are Leaders. The SEWA Movement in India, New Delhi.

Rowbotham, Sheila; Mitter, Swasti (eds.) (1994): Dignity and Daily Bread. New Forms of Economic Organising among Poor Women in the Third World and the first, New York.

Rowlands, Jo (1998): A Word of the Times, but What Does it Mean? Empowerment in the Discourse and Practice of Development, in: Afshar, Haleh (ed.): Women and Empowerment, New York, 11-35.

Scheu, Hildegard (1995): Entwicklungsziel: Frauenmacht! Frauenarbeit und Frauenorganisationen in Indien, Frankfurt.

Schneider, Ingrid (1988): Si, Senora. Hausangestellte in Kolumbien, in: Peripherie 30/31, 84-104.

Sebstad, Jennifer (1992): Struggle and Development among Self-Employed Women: A Report on SEWA, Washington.

Sen, Amartya (1982): Poverty and Famines. An Essay on Entitlement and Deprivation, Oxford.

- (1990): Gender and Cooperative Conflicts, in: Irene Tinker (ed.): Persistent Inequalities, Oxford, 123-149.

SEWA (1997): 25 years of SEWA Movement, Ahmedabad.

SEWU (1999): SEWU News, No 33, January 1999.

Schultz, Ulrike (1990): Die Last der Arbeit und der Traum vom Reichtum. Frauengruppen in Kenia zwischen gegenseitiger Hilfe und betriebswirtschaftlichem Kalkül, FU Berlin.

Südwind (1994): Bürsten gegen den Strich. Frauen im Reinigungsgewerbe, Siegburg.

Wichterich, Christa (1992): Moral, Markt, Macht. Frauengruppen in Kenia, in: Peripherie 47/48, S. 7-22.

Young, Kate (1993): Planning Development with Women: Making a World of Difference, London. 